\title{
Onychomatricoma: a tumor unknown to dermatologists*
}

\author{
Glaysson Tassara Tavares ${ }^{1}$ \\ Nilton Gioia Di Chiacchio ${ }^{2}$
}

\author{
Nilton Di Chiacchio² \\ Marcos Vilela de Souza ${ }^{1}$
}

DOI: $h$ ttp://dx.doi.org/10.1590/abd1806-4841.20153650

\begin{abstract}
A sixty-one year old white female was referred to the Dermatology Department to treat an ingrown nail in the inner corner of the left hallux. Examination of the entire nail unit showed the presence of xanthonychia in the outer corner besides thickening and increase in the transverse curvature of the nail plate. Dermoscopy and nuclear magnetic resonance of the free edge of the nail plate detected characteristic signs of onychomatricoma, a diagnosis that was later confirmed by anatomopathological exam.
\end{abstract}

Keywords: Nail diseases; Neoplasms; Onychomycosis

A sixty-one year old white female was referred to our service to treat an ingrown nail in the inner corner of the left hallux She reported a history of "alteration in nail color for the last 30 years, with subsequent episodes of ingrown nail, becoming worse in the last six months". During clinical examination, besides the presence of secretion, granulation tissue (detected only in the first consultation), erythema and pain, we observed an increase in the nail plate transverse curvature, causing the ingrowth (Figure 1). In the external corner of the same nail we noted the presence of xanthonychia, thickening of the nail plate, and heightened transverse curvature (Figure 2).

Dermoscopy of the free edge of the nail plate showed the presence of small perforations, limited to the site of the thickening (Figure 3).

Considering the above, an MRI was requested and revealed the presence of filament-like digitations protruding from the matrix (Figure 4). The patient underwent surgery with the diagnosis later confirmed by anatomopathological examination (Figure 5).

After a year of follow-up there was no sign of relapse or ungual dystrophy.
The onychomatricoma, first described by Baran and Kint in 1992, is a rare benign tumor of the nail matrix, characterized by having digitiform projections arising from the matrix. ${ }^{1,2}$ It is the only tumor in which the change of the nail plate has its origin in the matrix. Its etiology is not yet fully known. This tumor affects mostly women, with a peak of incidence in the $5^{\text {th }}$ decade of life and it is usually slow growing and painless.

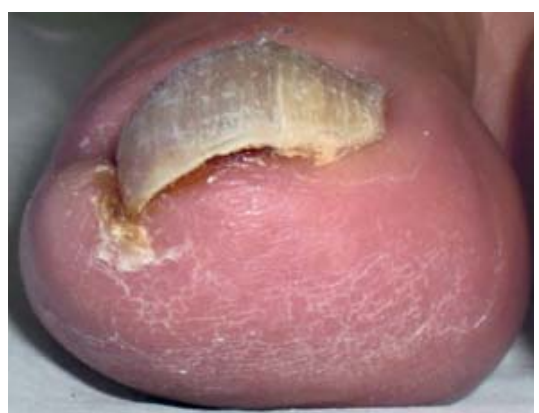

FIGURE 1: Ingrowth at the inner corner, presence of transverse over-curvature, ungual hyperkeratosis and xanthonychia 


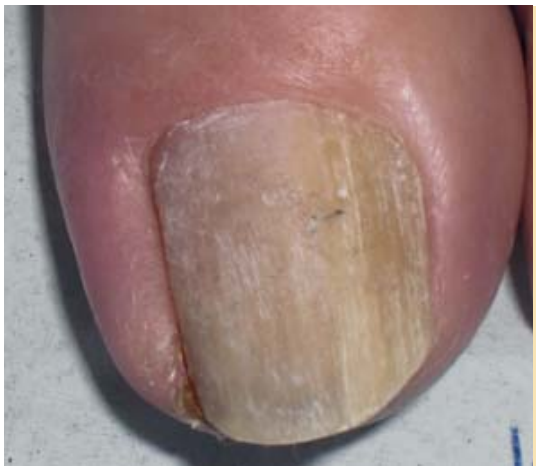

Figure 2: Xanthonychi a (longitudinal yellowish stripe), inner c o r $\mathrm{n}$ e $\mathrm{r}$ ing row th, and transverse over-curvature

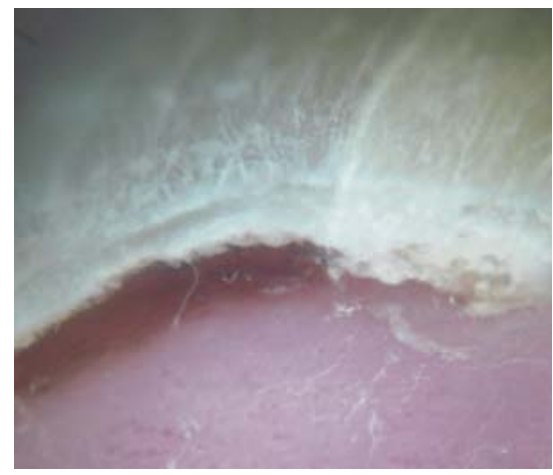

Figure 3: Dermoscopy the free ungual edge showing perforations on the distal portion of the nail plate (DermLite DL3 U.S.A.)

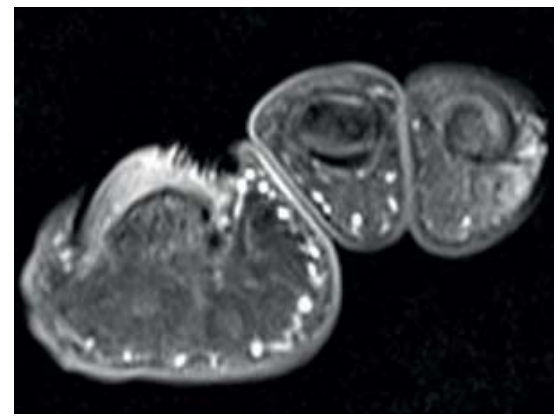

Figure 4: MRI (T2) showing fingerlike projections located on the lateral portion of the nail matrix

Classic presentation includes the clinical tetrad: xanthonychia, ungual hyperkeratosis, splinter hemorrhages affecting the nail plate, and longitudinal and transverse over-curvature of the nail plate. ${ }^{3}$ Besides the classic tetrad, the onychomatricoma can present as longitudinal melanonychia, nail dystrophy, subungual hematoma, nodule (elevation and erythema of the soft tissues), verrucosity of the proximal ungual fold and dorsal pterygium. Upon completion of the nail avulsion, the diagnosis becomes quite suggestive with the presence of digitiform projections.

The tumor is often confused with onychomycosis and treated as such. There is no report of onychomatricoma manifesting as ingrown toenail. The

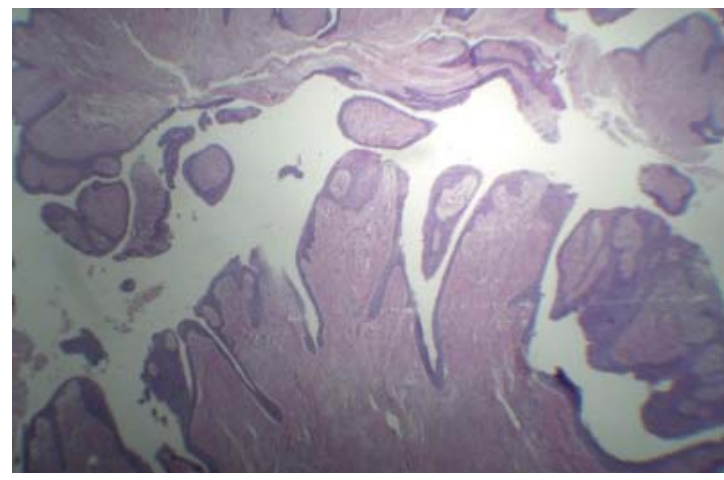

FIGURE 5: Histologic exam showing proliferation of the epidermis at the proximal fold/matrix, often coating papilliferous / digitiform structures, with a fibrous axis projecting on the nail plate (HE - 40x)

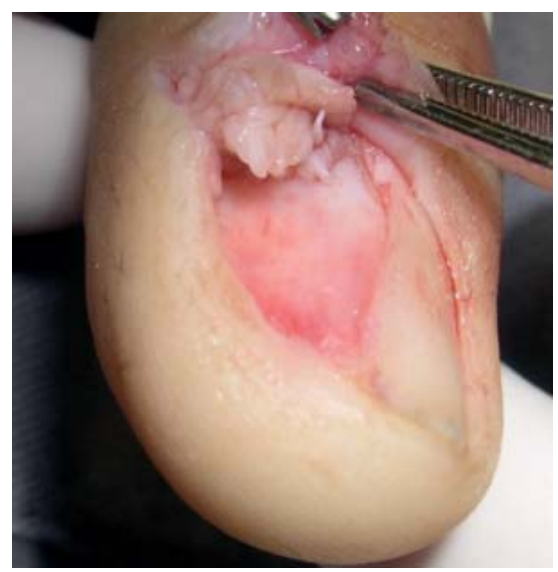

Figure $\quad 6$ : Intra-opera$t$ o $r \begin{array}{lll} & \mathrm{y} & \text {. }\end{array}$ Onychomatri coma with tumoral projections seen after the avulsion of the nail plate (illustrative image of another clinical case)

main differential diagnoses are: subungual exostosis, fibrokeratoma, fibroma, onychomycosis, squamous cell carcinoma, Bowen's disease, common wart, longitudinal melanonychia and osteochondroma.

Diagnosis is achieved through the classic tetrad signs, as well as additional diagnostic methods such as dermoscopy, ultrasound, magnetic resonance imaging (MRI), nail clipping and anatomopathological study. Dermoscopy shows perforations in the distal portion of the nail plate, hemorrhagic striae and white longitudinal ridges that correspond to the nail plate (Figure 3) furrows. ${ }^{4}$ Ultrasonography shows the tumoral lesion as a hypoechogenic area affecting the nail matrix and a hyperechogenic area corresponding to the digitiform (or fingerlike) projections. ${ }^{5}$ On MRI the portion that affects the nail matrix has a low signal uptake, while fingerlike, distal projections have a high 
uptake (Figure 4). ${ }^{6}$ Ungual clipping corresponds to the distal plate cut that is histologically evaluated, showing peculiar characteristics like thickening of the nail plate, with cavities filled with serous material and a thin layer of epithelium on its peripheral area. ${ }^{7}$ Histological study of the tumor resected from the matrix is considered as the gold standard for diagnosis (Figure 5). ${ }^{8}$
The treatment of onychomatricoma is surgical. After anesthetic block, the avulsion of the nail plate is performed and one can visualize the tumor projections, which will be removed with scissors or scalpel (Figure 6). The procedure is followed by wound dressing.

Results are good. However, depending on the extent of the tumor, some form of nail dystrophy can occur.

\section{REFERENCES}

1. Baran R, Klint A. Onychomatrixoma. Filamentous tufted tumor in the matrix of a funnel-shaped entity (report of three cases). Br J Dermatol. 1992;126:510-5.

2. Di Chiacchio N, Tavares GT, Padoveze EH, Bet DL, Di Chiacchio NG. Onicomatricoma Surg Cosmet Dermatol. 2013;5:10-4.

3. Perrin $\mathrm{CH}$, Goettmann S, Baran R. Onychomatricoma: clinical and histopathologic findings in 12 cases. J Am Acad Dermatol. 1998;39:560-4.

4. Di Chiacchio ND, Farias DC, Piraccini BM, Hirata SH, Richert B, Zaiac M, et al. Consensus on melanonychia nail plate dermoscopy. An Bras Dermatol. 2013;88:309-13

5. Soto R, Wortsman X, Corredoira Y. Onychomatricoma: clinical and sonographic findings. Arch Dermatol. 2009;145:1461-2.

6. Goetmann S, Drapé JL, Baran R, Perrin C, Haneke E, Belaïch S. Onychomatricome: 3 nouveaux cas: intérêt de la résonance magnétique nucléaire. Ann Dermatol Venereol. 1994;121:S145.

7. Miteva M, de Farias DC, Zaiac M, Romanelli P, Tosti A. Nail Clipping Diagnosis of Onychomatricoma. Arch Dermatol. 2011;147:1117-8.

8. Gaertner EM, Gordon M, Reed T. Onychomatricoma: case report of an unusual subungual tumor with literature review. J Cutan Pathol. 2009;36:66-9.

\author{
MAILING ADDRESS: \\ Marcos Vilela de Souza \\ Anexo Osvaldo Costa (Dermatologia) \\ Alameda Álvaro Celso, 155 \\ Santa Efigênia \\ 30130-100 - Belo Horizonte - MG \\ Brazil \\ E-mail:marcosviso@gmail.com
}

How to cite this article: Tavares GT, Di Chiacchio N, Di Chiacchio NG, Souza MV. Onychomatricoma: a tumor unknown to dermatologists. An Bras Dermatol. 2015;90(2):265-7. 